\title{
Limits on the Neutrino Velocity, Lorentz Invariance, and the Weak Equivalence Principle with TeV Neutrinos from Gamma-Ray Bursts
}

\author{
Jun-Jie Wei, ${ }^{a, b}$ Xue-Feng Wu, ${ }^{a, c}$ He Gao ${ }^{d}$ and Peter Mészáros ${ }^{e, f, g}$ \\ ${ }^{a}$ Purple Mountain Observatory, Chinese Academy of Sciences, Nanjing 210008, China \\ ${ }^{b}$ Guangxi Key Laboratory for Relativistic Astrophysics, Nanning 530004, China \\ ${ }^{c}$ Joint Center for Particle, Nuclear Physics and Cosmology, Nanjing University-Purple Moun- \\ tain Observatory, Nanjing 210008, China \\ ${ }^{d}$ Department of Astronomy, Beijing Normal University, Beijing 100875, China \\ ${ }^{e}$ Department of Astronomy and Astrophysics, Pennsylvania State University, 525 Davey \\ Laboratory, University Park, PA 16802 \\ ${ }^{f}$ Department of Physics, Pennsylvania State University, 104 Davey Laboratory, University \\ Park, PA 16802 \\ ${ }^{g}$ Center for Particle and Gravitational Astrophysics, Institute for Gravitation and the Cos- \\ mos, Pennsylvania State University, 525 Davey Laboratory, University Park, PA 16802 \\ E-mail: jjwei@pmo.ac.cn; xfwu@pmo.ac.cn; gaohe@bnu.edu.cn; nnp@psu.edu
}

\begin{abstract}
Five TeV neutrino events weakly correlated with five gamma-ray bursts (GRBs) were detected recently by IceCube. This work is an attempt to show that if the GRB identifications are verified, the observed time delays between the $\mathrm{TeV}$ neutrinos and gammaray photons from GRBs provide attractive candidates for testing fundamental physics with high accuracy. Based on the assumed associations between the TeV neutrinos and GRBs, we find that the limiting velocity of the neutrinos is equal to that of photons to an accuracy of $\sim 1.9 \times 10^{-15}-2.5 \times 10^{-18}$, which is about $10^{4}-10^{7}$ times better than the constraint obtained with the neutrino possibly from a blazar flare. In addition, we set the most stringent limits up to date on the energy scale of quantum gravity for both the linear and quadratic violations of Lorentz invariance, namely $E_{\mathrm{QG}, 1}>6.3 \times 10^{18}-1.5 \times 10^{21} \mathrm{GeV}$ and $E_{\mathrm{QG}, 2}>$ $2.0 \times 10^{11}-4.2 \times 10^{12} \mathrm{GeV}$, which are essentially as good as or are an improvement of one order of magnitude over the results previously obtained by the GeV photons of GRB 090510 and the PeV neutrino from a blazar flare. Assuming that the Shapiro time delay is caused by the gravitational potential of the Laniakea supercluster of galaxies, we also place the tightest limits to date on Einstein's weak equivalence principle through the relative differential variations of the parameterized post-Newtonian parameter $\gamma$ values for two different species of particles (i.e., neutrinos and photons), yielding $\Delta \gamma \sim 10^{-11}-10^{-13}$. However, it should be emphasized again that these limits here obtained are at best forecast of what could be achieved if the GRB/neutrino correlations would be finally confirmed.
\end{abstract}

Keywords: gamma ray burst experiments, ultra high energy photons and neutrinos, gravity 


\section{Contents}

1 Introduction $\quad 1$

2 Observational data $\quad 2$

3 New precision limits on fundamental physics with neutrinos from GRBs 3

3.1 Constraining the neutrino velocity 3

3.2 Constraining violation of the Lorentz invariance 4

3.3 Testing the weak equivalence principle 5

4 Summary and discussion

\section{Introduction}

The IceCube Collaboration recently performed a search for neutrinos of all flavors during the prompt emission of 807 gamma-ray bursts (GRBs) over the entire sky [1]. This search in three years of IceCube data resulted in five cascade neutrino candidate events correlated with five GRBs. These spatial and temporal coincidences yielded a combined P-value of 0.32, which was obtained from the total test statistic value with respect to the background-only distribution. In other words, the significance of these neutrino events being associated with GRBs is not high, and they are compatible with the background expectation. Nevertheless, it has been proposed that such associations between the high energy cosmic neutrinos and GRBs, if truly confirmed in the future, would be a powerful tool to constrain violations of the Lorentz invariance and equivalence principle (see e.g. [2-4]). We now discuss the significant capability of these five associations for testing fundamental physics, such as the neutrino velocity, Lorentz invariance, and the weak equivalence principle (WEP).

Recently, a theoretical constraint on superluminal neutrino velocity, $|v-c| / c \leq(0.5-$ $1.0) \times 10^{-20}$, has been obtained by treating kinematically allowed energy losses of superluminal neutrinos arising from vacuum pair emission and neutrino splitting [5]. The nearly simultaneous detection of neutrinos and light from astronomical sources provide a useful model-independent method for constraining the neutrino velocity. Using the arrival time delay of neutrinos and photons from supernova 1987A, Ref. [6] showed that the velocity of neutrinos is equal to that of photons to an accuracy of $\sim 2 \times 10^{-9}$. This result was further improved by [7] who used the same method and placed a limit on the relative velocity difference of $|v-c| / c \lesssim \mathcal{O}\left(10^{-11}\right)$ from an association between a $\mathrm{PeV}$ neutrino and the outburst activity of blazar PKS B1424-418.

Lorentz invariance is one of the fundamental principles of special relativity. However, some Quantum Gravity (QG) models predict that quantum fluctuations in the space-time would make it appear 'foamy' on short time and distance scales [8]. Thus, the propagation of massless particles (photons, or in the limit, neutrinos) through the foamy structure of space-time would exhibit a non-trivial dispersion relation in vacuo [9] which could lead to the violation of Lorentz invariance (LIV). Since it is typically expected for QG to manifest itself fully at the Planck scale, the QG energy scale $E_{\mathrm{QG}}$ is approximated to the Planck energy scale $E_{\mathrm{Pl}}$, i.e., $E_{\mathrm{QG}} \approx E_{\mathrm{Pl}}=\sqrt{\hbar c^{5} / G} \simeq 1.22 \times 10^{19} \mathrm{GeV}$ (see [10, 11], and references therein). Hence, $E_{\mathrm{Pl}}$ is a natural scale at which one expects Lorentz invariance to be broken. 
As a result of LIV effect, the speed of propagation of particles (neutrinos or photons) could become energy-dependent in vacuum (see e.g. $[9,12,13]$ ). The leading term in the modified dispersion relation is

$$
E^{2} \simeq p^{2} c^{2}+m^{2} c^{4} \pm E^{2}\left(\frac{E}{E_{\mathrm{QG}, \mathrm{n}}}\right)^{\mathrm{n}}
$$

where $E_{\mathrm{QG}}$ is the QG energy scale, $m$ is the rest-mass of the particle, the n-th order expansion of leading term corresponds to linear $(n=1)$ or quadratic $(n=2)$, and $+1(-1)$ stands for a decrease (increase) in particle speed with an increasing particle energy (also referred to as the "subluminal" and "superluminal" cases). The current best limits on the QG energy scale have been derived from the GeV photons of GRB 090510. The limits set are $E_{\mathrm{QG}, 1}>9.1 \times 10^{19}$ $\mathrm{GeV}>(1-10) E_{\mathrm{Pl}}$ and $E_{\mathrm{QG}, 2}>1.3 \times 10^{11} \mathrm{GeV}$ for linear and quadratic violations of Lorentz invariance, respectively $[14,15]$ (see also $[16,17]$ and summary constraints for Lorentz violation therein). Ref. [18] used the supernova 1987A neutrino data to study LIV effects, and obtained the limits of $E_{\mathrm{QG}, 1}>2.7 \times 10^{10} \mathrm{GeV}$ and $E_{\mathrm{QG}, 2}>4.6 \times 10^{4} \mathrm{GeV}$. Based on the association between the outburst of of the blazar PKS B1424-418 and a PeV neutrino, Ref. [4] set the most stringent limits up to now on neutrino LIV for subluminal neutrinos, implying $E_{\mathrm{QG}, 1}>1.1 \times 10^{17} \mathrm{GeV}$ and $E_{\mathrm{QG}, 2}>7.3 \times 10^{11} \mathrm{GeV}$.

Einstein's WEP is at the heart of general relativity as well as of other metric theories of gravity. The WEP states that any two different types of massless (or negligible rest-mass) messenger particles, or two of the same particles with different energies, if emitted simultaneously from the same astrophysical object and passing through the same gravitational field, should arrive at Earth at the same time [19,20]. The measurements of the arrival times of neutrinos and photons from supernova 1987A have been used [21, 22] to constrain the violations of the WEP through the Shapiro (gravitational) time delay effect [23]. They showed that the Shapiro delays of neutrino and photon are equal to an accuracy of approximately $0.34 \%$. With the flight time difference between the PeV neutrino and the blazar photons, Ref. [4] found that the constraints on the WEP accuracy from neutrinos can be further improved by two orders of magnitude when taking into account the gravitational potential of clusters or superclusters. Besides neutrinos, different-energy photons from extragalactic transients and variables have been applied to test the WEP through the Shapiro time delay effect, such as the photon emissions from GRBs [3, 24], fast radio bursts [25-28], and TeV blazars [29]. Moreover, such a test can now be extended to include a new messenger, namely gravitational waves $[30,31]$.

In this work, we determine the limits on the neutrino velocity, Lorentz invariance, and the WEP that would result if these five IceCube neutrinos were created in the same events as the gamma-ray photons observed in the associated GRBs.

\section{Observational data}

The IceCube Collaboration detected five neutrino events correlated with five GRBs [1]. Taking this association at face value, the data of these events and GRBs are shown in Table 1. The first eight columns include the following information for each association: (1) the source name; (2) the GRB prompt photon emission time $T_{100}$, which is defined by the most inclusive start and end times $\left(T_{1}\right.$ and $\left.T_{2}\right)$ reported by any satellite; (3) the observed maximum time delay $\Delta t$ between the start time of the GRB prompt photon emission and the arrival of the neutrino; the spatial parameters, including (4) the right ascension coordinate, (5) the 
declination coordinate; (6) the observed gamma-ray fluence of the GRBs; (7) the neutrino energy; and (8) the redshift.

The original idea of neutrino emission from GRBs [32] was that TeV neutrinos originate via interactions of shock-accelerated protons with the observed $\gamma$ rays $(p \gamma)$ in the same internal shocks which produce the $\mathrm{MeV}$ photons, i.e., they would be contemporaneous with the $\mathrm{MeV}$ photons. The same applies if the photons and neutrinos originate at the photospheric surface, e.g. [33]. Within the astrophysical model uncertainties, the difference between the proton and the electron acceleration times does not introduce an appreciable difference as far as the arrival times of the observable secondaries. The relativistic protons collide with photons produced by electrons in the same region where both protons and electrons were accelerated and where the photons were produced, so one does not expect a geometric delay. A delay of the neutrinos could be due to the neutrinos being produced in the sub-photosphere by $(p p)$ and $(p n)$ cascades, whose development would induce some delay of order seconds (see e.g. [34]). Another delay might be if the neutrinos originate from $(p \gamma)$ photohadronic processes in a different region than the $\mathrm{MeV}$ photons. E.g. the $\mathrm{MeV}$ photons might come from internal shocks, or a photosphere, while the neutrinos might come from the external reverse shock [35]. This would involve a geometric delay. However, in this case the neutrinos would be expected to be the $\mathrm{EeV}\left(10^{18} \mathrm{eV}\right)$ range, since the protons would be interacting with optical/ultraviolet photons, rather than with $\mathrm{MeV}$ photons. But the observed neutrinos are $\mathrm{TeV}$, so this is not a possibility.

In sum, it is reasonable to assume that the $\mathrm{TeV}$ neutrinos originate at the same time at which the GRB photon emission begins, or else that the neutrinos are observed of the order of seconds later than the start time of the GRB prompt emission. It should be underlined that our limits on fundamental physics are based on a relatively conservative estimate of the observed time delay, since we use the time interval between the start time of the GRB prompt emission and the arrival of the neutrino as the observed maximum time delay.

Except for GRB 101213A at $z=0.414$, the other four GRBs do not have measured redshifts. The empirical luminosity relation (the so-called Amati relation [36]) is therefore applied to estimate the redshift range of the four GRBs. We use the observed fluence, energy band, and spectral parameters of the four bursts ${ }^{1}$ to calculate the intrinsic isotropic gammaray energies and the intrinsic peak energies for different redshifts. By requiring that the bursts enter the $3 \sigma$ region of the relation, we derive $z \geq 0.170$ for GRB $110101 \mathrm{~B}, z \geq 0.237$ for GRB 110521B, $z \geq 0.269$ for GRB 111212A, and $z \geq 0.197$ for GRB 120114A (the lower limits of redshifts are conservatively adopted in the rest of this paper). Regarding the details on estimating the redshifts of GRBs, see [37] for a more detailed description. Here we adopt the cosmological parameters derived from the Planck observations [38]: $\Omega_{\mathrm{m}}=0.315$, $\Omega_{\Lambda}=0.685$, and $H_{0}=67.3 \mathrm{~km} \mathrm{~s}^{-1} \mathrm{Mpc}^{-1}$.

\section{New precision limits on fundamental physics with neutrinos from GRBs}

\subsection{Constraining the neutrino velocity}

Assuming the physical associations between the TeV neutrinos and GRBs, observational constraints on the neutrino velocity can be obtained. The simplest parametrization of the constraint is in terms of an effective limiting velocity of neutrinos $v$ compared to that of

\footnotetext{
${ }^{1}$ The relevant observed fluence, energy band, and spectral parameters of GRBs are available on the GRBweb database at http://grbweb.icecube.wisc.edu.
} 
Table 1. Limits on $|v-c| / c, E_{\mathrm{QG}, 1}, E_{\mathrm{QG}, 2}$, and $\Delta \gamma$ from the associations between the TeV neutrinos and GRBs.

\begin{tabular}{|c|c|c|c|c|c|c|c|c|c|c|c|}
\hline GRB/Neutrino & $\begin{array}{c}T_{100} \\
\text { (s) }\end{array}$ & $\begin{array}{l}\Delta t \\
(\mathrm{~s})\end{array}$ & $\begin{array}{l}\text { R.A.a } \\
(\operatorname{deg})\end{array}$ & $\begin{array}{l}\begin{array}{l}\text { Dec. } \\
(\mathrm{deg})\end{array}\end{array}$ & $\begin{array}{c}\text { Fluence } \\
\left(\mathrm{erg} \mathrm{cm}^{-2}\right)\end{array}$ & $\begin{array}{c}\text { Energy } \\
(\mathrm{TeV})\end{array}$ & $\overline{\bar{z}}$ & $\mid \overrightarrow{|v-c| / c}$ & $\begin{array}{c}E_{\mathrm{QG}, 1} \\
(\mathrm{GeV})\end{array}$ & $\begin{array}{l}E_{\mathrm{QG}, 2} \\
(\mathrm{GeV})\end{array}$ & $\overline{\Delta \gamma \gamma}$ \\
\hline 101213A/Event 1 & 202 & 109 & 241.314 & 21.897 & $7.4 \times 10^{-6}$ & 11 & 0.414 & $6.4 \times 10^{-16}$ & $2.1 \times 10^{19}$ & $6.4 \times 10^{11}$ & $5.6 \times 10^{-11}$ \\
\hline 110101B/Event 2 & 235 & 141 & 105.500 & 34.580 & $6.6 \times 10^{-6}$ & 34 & $0.170^{b}$ & $1.9 \times 10^{-15}$ & $2.0 \times 10^{19}$ & $1.0 \times 10^{12}$ & $9.6 \times 10^{-11}$ \\
\hline $110521 \mathrm{~B} /$ Event 3 & 6.14 & 0.26 & 57.540 & -62.340 & $3.6 \times 10^{-6}$ & 3.4 & $0.237^{b}$ & $2.5 \times 10^{-18}$ & $1.5 \times 10^{21}$ & $2.9 \times 10^{12}$ & $1.3 \times 10^{-13}$ \\
\hline $111212 \mathrm{~A} /$ Event 4 & 68.5 & 11.7 & 310.431 & -68.613 & $1.4 \times 10^{-6}$ & 30.6 & $0.269^{b}$ & $1.0 \times 10^{-16}$ & $3.4 \times 10^{20}$ & $4.2 \times 10^{12}$ & $6.0 \times 10^{-12}$ \\
\hline $120114 \mathrm{~A} /$ Event 5 & 43.3 & 57.2 & 317.904 & 57.036 & $2.4 \times 10^{-6}$ & 3.8 & $0.197^{b}$ & $6.7 \times 10^{-16}$ & $6.3 \times 10^{18}$ & $2.0 \times 10^{11}$ & $1.9 \times 10^{-11}$ \\
\hline
\end{tabular}

photons $c$. This limit on the relative velocity difference will be [39]

$$
|v-c| / c \leq c \cdot \Delta t / D
$$

where $D=\frac{c}{H_{0}} \int_{0}^{z} \frac{d z^{\prime}}{\sqrt{\Omega_{m}\left(1+z^{\prime}\right)^{3}+\Omega_{\Lambda}}}$ is the co-moving distance of the source. The limits on $|v-c| / c$ for each event are presented in column 9 of Table 1 . The strictest limit is $\mid v-$ $c \mid / c \leq 2.5 \times 10^{-18}$ for GRB $110521 \mathrm{~B} /$ Event 3 and the worst limit is $1.9 \times 10^{-15}$ for GRB $110101 \mathrm{~B} /$ Event 2, which are close to $10^{4}-10^{7}$ times better than the neutrino-velocity limit obtained with the neutrino from a blazar flare [7].

\subsection{Constraining violation of the Lorentz invariance}

Note that the superluminal neutrinos would loss their energy quickly due to both vacuum pair emission and neutrino splitting [5, 40], and some excellent limits on LIV have been derived from superluminal neutrinos [41-43]. Here we set the limits on LIV for subluminal neutrinos, i.e., the low energy photons travel faster than the high energy neutrinos. As the test particles are massless or nearly massless, the term $m^{2} c^{4}$ in Equation (1.1) is absolutely negligible. Since the speed of particles have an energy dependence, two particles with different energies emitted simultaneously from the source will arrive at the observer with a time delay $\Delta t$. For a cosmic transient source, one has to consider the cosmological expansion when calculating the LIV induced time delay (see e.g. [18, 44-47])

$$
\Delta t=\frac{1+\mathrm{n}}{2 H_{0}} \frac{E_{h}^{\mathrm{n}}-E_{l}^{\mathrm{n}}}{E_{\mathrm{QG}, \mathrm{n}}^{\mathrm{n}}} \int_{0}^{z} \frac{\left(1+z^{\prime}\right)^{\mathrm{n}} d z^{\prime}}{\sqrt{\Omega_{m}\left(1+z^{\prime}\right)^{3}+\Omega_{\Lambda}}},
$$

where $E_{h}$ and $E_{l}\left(E_{h}>E_{l}\right)$ correspond to the different particle energies.

The limits on $E_{\mathrm{QG}, 1}$ and $E_{\mathrm{QG}, 2}$ for each GRB neutrino are presented in columns 10 and 11 of Table $1 .^{2}$ The tightest limits on the linear and quadratic terms are $E_{\mathrm{QG}, 1}>$ $1.5 \times 10^{21} \mathrm{GeV}>100 E_{\mathrm{Pl}}$ for GRB $110521 \mathrm{~B} /$ Event 3 and $E_{\mathrm{QG}, 2}>4.2 \times 10^{12} \mathrm{GeV}$ for GRB $111212 \mathrm{~A} /$ Event 4 , respectively. The worst limits are $E_{\mathrm{QG}, 1}>6.3 \times 10^{18} \mathrm{GeV}$ and $E_{\mathrm{QG}, 2}>$ $2.0 \times 10^{11} \mathrm{GeV}$ for GRB $120114 \mathrm{~A} /$ Event 5 . We see that the linear $(\mathrm{n}=1) \mathrm{LIV}$ term can be easily excluded by GRB 110521B/Event 3. Compared with the previous best limit on $E_{\mathrm{QG}, 1}$ from $\mathrm{GeV}$ photons from $\mathrm{GRB} 090510\left(E_{\mathrm{QG}, 1}>9.1 \times 10^{19} \mathrm{GeV}[14]\right)$, our tightest limit on $E_{\mathrm{QG}, 1}$ represents an improvement of at least one order of magnitude, although our worst limit on $E_{\mathrm{QG}, 1}$ is not as stringent as that of GRB 090510. Moreover, our limits on $E_{\mathrm{QG}, 2}$ are as good as or even 10 times tighter than the previous best result from the blazar PeV neutrino $\left(E_{\mathrm{QG}, 2}>7.3 \times 10^{11} \mathrm{GeV}[4]\right)$. In short, we set the most stringent limits on both the

\footnotetext{
${ }^{2}$ Note that $E_{h}^{\mathrm{n}}-E_{l}^{\mathrm{n}}$ in Equation (3.2) can be approximated as $E_{h}^{\mathrm{n}}$, since the energies of GRB photons at the trigger time $(\sim 100 \mathrm{keV})$ are several orders of magnitude lower than those of neutrinos $(\sim \mathrm{TeV})$.
} 
linear and quadratic LIV effects to date under the assumption that the associations between the $\mathrm{TeV}$ neutrinos and GRBs would be finally confirmed.

Here we want to point out a possible caveat to the $\mathrm{n}=1$ limits. In an effective field theory framework, the dispersion relation (see Equation 1.1) for $\mathrm{n}=1$ would imply CPT odd terms which predict opposite signs for the Lorentz breaking term for neutrino and antineutrino. In other words, if the neutrino is e.g. superluminal, then the antineutrino will be subluminal. However, the IceCube detector does not distinguish neutrinos from antineutrinos. So for an initial equal amounts of neutrino and antineutrino, one expect that superluminal neutrinos will probably decay in lower energy neutrinos and antineutrinos with the latter having partially travel faster than expected. It is hard to know whether the two effects can compensate each other or not. If not, this would add another error term to the observed time delay and affect our purposed constraints at some level.

\subsection{Testing the weak equivalence principle}

Indeed, all metric theories of gravity satisfying the WEP predict that all test particles must follow same trajectories and undergo the identical Shapiro time delay. That is, as long as the WEP is valid, all metric theories expect $\gamma_{1}=\gamma_{2} \equiv \gamma$, where $\gamma$ is the parametrized post-Newtonian (PPN) parameter and the subscripts correspond to two different particles $[19,20,48]$. The WEP accuracy can therefore be described by limits on the differences of $\gamma$ values for different particles. According to the Shapiro time delay effect [23], the time interval for particles to traverse a given distance is longer by

$$
t_{\mathrm{gra}}=-\frac{1+\gamma}{c^{3}} \int_{r_{e}}^{r_{o}} U(r) d r
$$

in the presence of a gravitational potential $U(r)$, where the integration is along the path of the particle emitted at $r_{e}$ and received at $r_{o}$. If the values of the PPN parameter $\gamma$ are different for different particles, two particles emitted simultaneously from the source will reach us at different times, and the corresponding time delay is given by

$$
\Delta t_{\text {gra }}=\frac{\gamma_{1}-\gamma_{2}}{c^{3}} \int_{r_{o}}^{r_{e}} U(r) d r,
$$

where $\gamma_{1}-\gamma_{2}$ represents the difference between the $\gamma$ values for different particles.

To calculate the Shapiro time delay with Equation (3.4), we need to know the gravitational potential $U(r)$. Most previous studies focused on the contribution from the gravitational potential of the Milky Way. However, it has been showed that incorporating the gravitational potential of the large scale structure would tighten the constraints on the WEP accuracy $[4,26,27,49]$. We here consider the gravitational potential of the Laniakea supercluster of galaxies. Laniakea is a newly discovered supercluster of galaxies, in which the Milky Way as well as the Local Group reside [50]. The total mass of Laniakea is $10^{17} M_{\odot}$, which is about $10^{5}$ times heavier than the Milky Way.

Assuming that the measured time delays $(\Delta t)$ between correlated particles from the same source are mainly caused by the gravitational potential of the Laniakea supercluster of 
Table 2. Limits on $|v-c| / c, E_{\mathrm{QG}, 1}, E_{\mathrm{QG}, 2}$, and $\Delta \gamma$ using the GRB durations $T_{100}$ as the conservative limits of the observed time delays.

\begin{tabular}{|c|c|c|c|c|c|c|c|c|c|c|c|}
\hline GRB/Neutrino & $\begin{array}{c}T_{100} \\
(\mathrm{~s})\end{array}$ & $\begin{array}{ll}\Delta t \\
(\mathrm{~s})\end{array}$ & $\begin{array}{l}\text { R.A. } \\
\text { (deg) }\end{array}$ & $\begin{array}{l}\text { Dec. } \\
\text { (deg) }\end{array}$ & $\begin{array}{c}\text { Fluence } \\
\left(\text { erg cm }^{-2}\right)\end{array}$ & $\begin{array}{l}\begin{array}{l}\text { Energy } \\
(\mathrm{TeV})\end{array} \\
\end{array}$ & $\overline{\bar{z}}$ & $\overline{|| v-c \mid / c}$ & $\begin{array}{l}E_{\mathrm{QG}, 1} \\
(\mathrm{GeV})\end{array}$ & $\begin{array}{l}E_{\mathrm{QG}, 2} \\
(\mathrm{GeV}) \\
\end{array}$ & $\overline{\overline{\Delta \gamma \gamma}}$ \\
\hline 101213A/Event 1 & 202 & 109 & 241.314 & 21.897 & $7.4 \times 10^{-6}$ & 11 & 0.414 & $1.2 \times 10^{-15}$ & $1.1 \times 10^{19}$ & $4.7 \times 10^{11}$ & $1.0 \times 10^{-10}$ \\
\hline 110101B/Event 2 & 235 & 141 & 105.500 & 34.580 & $6.6 \times 10^{-6}$ & 34 & 0.170 & $3.1 \times 10^{-15}$ & $1.2 \times 10^{19}$ & $8.1 \times 10^{11}$ & $1.6 \times 10^{-10}$ \\
\hline $110521 \mathrm{~B} /$ Event 3 & 6.14 & 0.26 & 57.540 & -62.340 & $3.6 \times 10^{-6}$ & 3.4 & 0.237 & $6.0 \times 10^{-17}$ & $6.3 \times 10^{19}$ & $6.0 \times 10^{11}$ & $3.0 \times 10^{-12}$ \\
\hline 111212A/Event 4 & 68.5 & 11.7 & 310.431 & -68.613 & $1.4 \times 10^{-6}$ & 30.6 & 0.269 & $5.9 \times 10^{-16}$ & $5.8 \times 10^{19}$ & $1.7 \times 10^{12}$ & $3.5 \times 10^{-11}$ \\
\hline $120114 \mathrm{~A} /$ Event $5^{a}$ & 43.3 & 57.2 & 317.904 & 57.036 & $2.4 \times 10^{-6}$ & 3.8 & 0.197 & $6.7 \times 10^{-16}$ & $6.3 \times 10^{18}$ & $2.0 \times 10^{11}$ & $1.9 \times 10^{-11}$ \\
\hline
\end{tabular}

galaxies, and adopting a Keplerian potential for Laniakea ${ }^{3}$, we have [22]

$$
\begin{aligned}
\Delta t> & \Delta t_{\text {gra }}=\left(\gamma_{1}-\gamma_{2}\right) \frac{G M_{\mathrm{L}}}{c^{3}} \times \\
& \ln \left\{\frac{\left[d+\left(d^{2}-b^{2}\right)^{1 / 2}\right]\left[r_{L}+s_{\mathrm{n}}\left(r_{L}^{2}-b^{2}\right)^{1 / 2}\right]}{b^{2}}\right\},
\end{aligned}
$$

where $M_{\mathrm{L}} \simeq 10^{17} M_{\odot}$ is the Laniakea mass [50], $d$ denotes the distance from the source to the Laniakea center (for a cosmic source, $d$ is approximated as the distance from the source to the Earth), $b$ represents the impact parameter of the particle paths relative to the Laniakea center, and $s_{\mathrm{n}}= \pm 1$ corresponds to the sign of the correction of the source direction. If $s_{\mathrm{n}}=+1\left(s_{\mathrm{n}}=-1\right)$, the source is located along the direction of Laniakea (anti-Laniakea) center. Since the gravitational center of Laniakea is considered to be the Great Attractor [51], a mass concentration in the nearby Universe, we adopt the coordinates of the Great Attractor center $\left(\right.$ R.A. $=10^{\mathrm{h}} 32^{\mathrm{m}}$, Dec. $\left.=-46^{\circ} 00^{\prime}\right)$ instead of that of the Laniakea center. For a cosmic source in the position $\left(\right.$ R.A. $=\beta_{\mathrm{s}}$, Dec. $=\delta_{\mathrm{s}}$ ), the impact parameter can be written as

$$
b=r_{L} \sqrt{1-\left(\sin \delta_{s} \sin \delta_{L}+\cos \delta_{s} \cos \delta_{L} \cos \left(\beta_{s}-\beta_{L}\right)\right)^{2}},
$$

where $r_{L}=79 \mathrm{Mpc}$ is the distance from the Earth to the center of Laniakea, and $\left(\beta_{L}=\right.$ $\left.10^{\mathrm{h}} 32^{\mathrm{m}}, \delta_{L}=-46^{\circ} 00^{\prime}\right)$ represent the coordinates of the Laniakea center.

Thus, with the observed time delays between the beginning of the GRB prompt photon emission and the arrival of the neutrinos in Table 1, we obtain WEP constraints from Equation (3.6) for assuming that these five TeV neutrinos are truly associated with GRBs. The limits on $\gamma_{\nu}-\gamma_{\gamma}$ for each event are displayed in column 12 of Table 1 . The strictest limit is $\gamma_{\nu}-\gamma_{\gamma}<1.3 \times 10^{-13}$ for GRB 110521B/Event 3 and the worst limit is $9.6 \times 10^{-11}$ for GRB 110101B/Event 2, which are about $5-7$ orders of magnitude more constraining than the previous limit obtained with the PeV neutrino from a blazar flare $\left(\gamma_{\nu}-\gamma_{\gamma}<7.0 \times 10^{-6}\right.$ $[4])$.

\section{Summary and discussion}

In this work, we discuss the potential of five possible GRB/neutrino associations from the IceCube neutrino observatory for probing fundamental physics. We show that if the GRB

\footnotetext{
${ }^{3}$ In fact, the gravitational potential of the Laniakea supercluster is still not well known. Besides the Keplerian potential model, Ref. [21] also examined the other widely used potential model, i.e., the isothermal potential. They showed that these two different potential models do not have a strong influence on the results for testing the WEP. Here we adopt the Keplerian potential for the whole supercluster.
} 
identifications are verified, significant improvements can be obtained on limits on the neutrino velocity, the violation of Lorentz invariance, and the accuracy of the WEP, by using the observed time delays between the neutrinos and photons. First, the strictest limit on the relative velocity difference is $|v-c| / c \leq 2.5 \times 10^{-18}$ for GRB $11052 \mathrm{~B} /$ Event 3, and the worst limit still comes to $1.9 \times 10^{-15}$ for GRB $110101 \mathrm{~B} /$ Event 2, which are about $10^{4}-10^{7}$ times better than the neutrino-velocity limit obtained with the neutrino from a blazar flare. Secondly, we place the most stringent limits to date on both the linear and quadratic LIV terms, namely $E_{\mathrm{QG}, 1}>6.3 \times 10^{18}-1.5 \times 10^{21} \mathrm{GeV}$ and $E_{\mathrm{QG}, 2}>2.0 \times 10^{11}-4.2 \times 10^{12}$ $\mathrm{GeV}$, which are as good as or are an improvement of one order of magnitude over the results previously obtained from the GeV photons of GRB 090510 and the blazar PeV neutrino. Finally, we find that the limits on the differences of the $\gamma$ values for the neutrinos and photons are as low as $\sim 10^{-11}-10^{-13}$, improving the limits on the WEP from the blazar/neutrino association by at least $5-7$ orders of magnitude. Nevertheless, it should be underlined that these limits are at best forecast of what could be achieved if the GRB/neutrino associations would be finally verified.

As described above, for a GRB/neutrino association, the neutrinos would be contemporaneous with the $\mathrm{MeV}$ photons. We have first set a relatively conservative limit by using the time interval between the start time of the GRB prompt emission and the arrival of the neutrino as the observed time delay when testing fundamental physics. However, the neutrino and photon production is stochastic (and much fewer neutrinos are produced than photons), so the first neutrino could have been produced well after the first photon (but before the last photon, if the same shocks that accelerate electrons radiating photons are also responsible for accelerating the protons which produce neutrinos). That is, the observed time delay of any neutrino and photon in the association system couldn't be longer than the GRB prompt photon emission time $\left(T_{100}\right)$. To account for the uncertainty of the observed time delay, we also test one more case by assuming $T_{100}$ as the conservative limit of the observed time delay. The more conservative limits on $|v-c| / c, E_{\mathrm{QG}, 1}, E_{\mathrm{QG}, 2}$, and $\Delta \gamma$ are shown in Table 2. One can see from this table that the implications of the GRB neutrino tests of fundamental physics are not greatly affected. Compared with those constraints presented in Table 1, the new constraint results only vary within a factor of two to 1 order of magnitude.

It has long been proposed that high energy neutrinos would be associated with GRBs. Thanks to the great wide field of view and high sensitivity, very-large-volume neutrino telescopes such as ANTARES and IceCube are ideal detectors to search for any high-energy neutrinos from GRBs, e.g. [52, 53]. Although the dedicated searches for high energy neutrinos correlated with GRBs have led to null results [54, 55], it is entirely possible that some of the IceCube neutrinos originate from GRBs, such as these five spatial and temporal coincidences for the neutrinos and GRBs [1]. If in the future the origin of these IceCube neutrinos is better understood, or the associations between different detected events are truly confirmed, the prospects for testing fundamental physics with high energy cosmic neutrinos, as discussed in this work, will be very promising.

\section{Acknowledgments}

We are grateful to the anonymous referee for the helpful suggestions which have helped us to improve the presentation of the paper. We also thank Lijing Shao and Xiang-Yu Wang for the useful communications. This work is partially supported by the National Basic Research Program ("973" Program) of China (Grants 2014CB845800 and 2013CB834900), 
the National Natural Science Foundation of China (grants Nos. 11322328, 11433009, and 11543005), the Natural Science Foundation of Jiangsu Province (Grant No. BK20161096), the Youth Innovation Promotion Association (2011231), the Strategic Priority Research Program "The Emergence of Cosmological Structures" (Grant No. XDB09000000) of the Chinese Academy of Sciences, the Guangxi Key Laboratory for Relativistic Astrophysics, and NASA NNX13AH50G.

\section{References}

[1] IceCube Collaboration, M. G. Aartsen, K. Abraham, M. Ackermann, J. Adams, J. A. Aguilar, et al., An All-Sky Search for Three Flavors of Neutrinos from Gamma-Ray Bursts with the IceCube Neutrino Observatory, arXiv:1601.06484.

[2] Jacob, U. \& T. Piran, Neutrinos from gamma-ray bursts as a tool to explore quantum-gravity-induced Lorentz violation, Nature Physics 3 (2007) 87, [arXiv:hep-ph/0607145].

[3] Gao, H., X.-F. Wu, \& P. Mészáros, Cosmic Transients Test Einstein's Equivalence Principle out to GeV Energies, ApJ 810 (2015) 121, [arXiv:1509.00150].

[4] Wang, Z.-Y., R.-Y. Liu, \& X.-Y. Wang, Testing the Equivalence Principle and Lorentz Invariance with PeV Neutrinos from Blazar Flares, Phys. Rev. Lett. 116 (2016) 151101, [arXiv:1602.06805].

[5] Stecker, F. W., S. T. Scully, S. Liberati, \& D. Mattingly, Searching for traces of Planck-scale physics with high energy neutrinos, Phys. Rev. D 91 (2015) 045009, [arXiv:1411.5889].

[6] Longo, M. J., Tests of relativity from SN1987A, Phys. Rev. D 36 (1987) 3276.

[7] Kadler, M., F. Krauß, K. Mannheim, R. Ojha, C. Müller, R. Schulz, et al., Coincidence of a high-fluence blazar outburst with a PeV-energy neutrino event, Nature Physics, in press, arXiv:1602.02012.

[8] Amelino-Camelia, G., J. Ellis, N. E. Mavromatos, \& D. V. Nanopoulos, Distance Measurement and Wave Dispersion in a Liouville-String Approach to Quantum Gravity, International Journal of Modern Physics A 12 (1997) 607, [arXiv:hep-th/9605211].

[9] Amelino-Camelia, G., J. Ellis, N. E. Mavromatos, D. V. Nanopoulos, \& S. Sarkar, Tests of quantum gravity from observations of $\gamma$-ray bursts, Nature 393 (1998) 763, [arXiv:astro$\mathrm{ph} / 9712103]$.

[10] Mattingly, D., Modern Tests of Lorentz Invariance, Living Reviews in Relativity 8 (2005) [arXiv:gr-qc/0502097].

[11] Amelino-Camelia, G., Quantum-Spacetime Phenomenology, Living Reviews in Relativity 16 (2013) [arXiv:0806.0339].

[12] Choubey, S. \& S. F. King, Electrophobic Lorentz invariance violation for neutrinos and the see-saw mechanism, Phys. Lett. B 586 (2004) 353, [arXiv:hep-ph/0311326].

[13] Ellis, J. \& N. E. Mavromatos, Probes of Lorentz violation, Astroparticle Physics 43 (2013) 50, [arXiv:1111.1178].

[14] Abdo, A. A., M. Ackermann, M. Ajello, K. Asano, W. B. Atwood, M. Axelsson, et al., A limit on the variation of the speed of light arising from quantum gravity effects, Nature 462 (2009) 331, [arXiv:0908.1832].

[15] Vasileiou, V., A. Jacholkowska, F. Piron, J. Bolmont, C. Couturier, J. Granot, et al., Constraints on Lorentz invariance violation from Fermi-Large Area Telescope observations of gamma-ray bursts, Phys. Rev. D 87 (2013) 122001, [arXiv:1305.3463]. 
[16] Kostelecký, V. A. \& N. Russell, Data tables for Lorentz and CPT violation, Reviews of Modern Physics 83 (2011) 11, [arXiv:0801.0287].

[17] Liberati, S., Tests of Lorentz invariance: a 2013 update, Classical and Quantum Gravity 30 (2013) 133001, [arXiv:1304.5795].

[18] Ellis, J., N. Harries, A. Meregaglia, A. Rubbia, \& A. S. Sakharov, Probes of Lorentz violation in neutrino propagation, Phys. Rev. D 78 (2008) 033013, [arXiv:0805.0253].

[19] Will, C. M., The Confrontation between General Relativity and Experiment, Living Reviews in Relativity 9 (2006) [arXiv:gr-qc/0510072].

[20] Will, C. M., The Confrontation between General Relativity and Experiment, Living Reviews in Relativity 17 (2014) [arXiv:1403.7377].

[21] Krauss, L. M. \& S. Tremaine, Test of the weak equivalence principle for neutrinos and photons, Phys. Rev. Lett. 60 (1988) 176.

[22] Longo, M. J., New precision tests of the Einstein equivalence principle from SN1987A, Phys. Rev. Lett. 60 (1988) 173.

[23] Shapiro, I. I., Fourth Test of General Relativity, Phys. Rev.. Lett. 13 (1964) 789.

[24] Sivaram, C., Constraints on the photon mass and charge and test of equivalence principle from GRB 990123, Bulletin of the Astronomical Society of India 27 (1999) 627.

[25] Wei, J.-J., H. Gao, X.-F. Wu, \& P. Mészáros, Testing Einstein's Equivalence Principle With Fast Radio Bursts, Phys. Rev. Lett. 115 (2015) 261101, [arXiv:1512.07670].

[26] Nusser, A., On Testing the Equivalence Principle with Extragalactic Bursts, ApJL $\mathbf{8 2 1}$ (2016) L2, [arXiv:1601.03636].

[27] Zhang, S.-N., Testing Einstein's Equivalence Principle with Cosmological Fast Radio Bursts behind Clusters of Galaxies, arXiv:1601.04558.

[28] Tingay, S. J. \& D. L. Kaplan, Limits on Einstein's Equivalence Principle from the First Localized Fast Radio Burst FRB 150418, ApJL 820 (2016) L31, [arXiv:1602.07643].

[29] Wei, J.-J., J.-S. Wang, H. Gao, \& X.-F. Wu, Tests of the Einstein Equivalence Principle Using TeV Blazars, ApJL 818 (2016) L2, [arXiv:1601.04145].

[30] Wu, X.-F., H. Gao, J.-J. Wei, P. Mészáros, B. Zhang, et al., Testing Einstein's Weak Equivalence Principle With Gravitational Waves, Phys. Rev. D 94 (2016) 024061, [arXiv:1602.01566].

[31] Kahya, E. O. \& S. Desai, Constraints on frequency-dependent violations of Shapiro delay from GW150914, Phys. Lett. B 756 (2016) 265, [arXiv:1602.04779].

[32] Waxman, E. \& J. Bahcall, High Energy Neutrinos from Cosmological Gamma-Ray Burst Fireballs, Phys. Rev. Lett. 78 (1997) 2292, [arXiv:astro-ph/9701231].

[33] Gao, S., K. Asano, \& P. Mészáros, High energy neutrinos from dissipative photospheric models of gamma ray bursts, JCAP 11 (2012) 058, [arXiv:1210.1186].

[34] Asano, K. \& P. Mészáros, Photon and neutrino spectra of time-dependent photospheric models of gamma-ray bursts, JCAP 9 (2013) 008, [arXiv:1308.3563].

[35] Waxman, E. \& J. N. Bahcall, Neutrino Afterglow from Gamma-Ray Bursts: 10 ${ }^{18}$ EV, ApJ 541 (2000) 707, [arXiv:hep- ph/9909286].

[36] Amati, L., F. Frontera, M. Tavani, J. J. M. in’t Zand, A. Antonelli, E. Costa, et al., Intrinsic spectra and energetics of BeppoSAX Gamma-Ray Bursts with known redshifts, A\&A 390 (2002) 81, [arXiv:astro-ph/0205230].

[37] Deng, W. \& B. Zhang, Cosmological Implications of Fast Radio Burst/Gamma-Ray Burst 
Associations, ApJL 783 (2014) L35, [arXiv:1401.0059].

[38] Planck Collaboration, P. A. R. Ade, N. Aghanim, C. Armitage-Caplan, M. Arnaud, M. Ashdown, et al., Planck 2013 results. XVI. Cosmological parameters, A\&A 571 (2014) A16, [arXiv:1303.5076].

[39] Schaefer, B. E., Severe Limits on Variations of the Speed of Light with Frequency, Phys. Rev. Lett. 82 (1999) 4964, [arXiv:astro-ph/9810479].

[40] Maccione, L., S. Liberati, \& D. M. Mattingly, Violations of Lorentz invariance in the neutrino sector: an improved analysis of anomalous threshold constraints, JCAP 3 (2013) 039.

[41] Borriello, E., S. Chakraborty, A. Mirizzi, \& P. D. Serpico, Stringent constraint on neutrino Lorentz invariance violation from the two IceCube PeV neutrinos, Phys. Rev. D 87 (2013) 116009, [arXiv:1303.5843].

[42] Díaz, J. S., V. A. Kostelecký, \& M. Mewes, Testing relativity with high-energy astrophysical neutrinos, Phys. Rev. D 89 (2014) 043005, [arXiv:1308.6344].

[43] Stecker, F. W., Limiting superluminal electron and neutrino velocities using the 2010 Crab Nebula flare and the IceCube PeV neutrino events, Astroparticle Physics 56 (2014) 16, [arXiv:1306.6095].

[44] Jacob, U. \& T. Piran, Lorentz-violation-induced arrival delays of cosmological particles, JCAP 1 (2008) 031, [arXiv:0712.2170].

[45] Shao, L., Z. Xiao, \& B.-Q. Ma, Lorentz violation from cosmological objects with very high energy photon emissions, Astroparticle Physics 33 (2010) 312, [arXiv:0911.2276].

[46] Chang, Z., Y. Jiang, \& H.-N. Lin, A unified constraint on the Lorentz invariance violation from both short and long GRBs, Astroparticle Physics 36 (2012) 47, [arXiv:1201.3413].

[47] Zhang, S. \& B.-Q. Ma, Lorentz violation from gamma-ray bursts, Astroparticle Physics 61 (2015) 108, [arXiv:1406.4568].

[48] Jacobson, T., \& D. Mattingly, Gravity with a dynamical preferred frame, Phys. Rev. D 64 (2001) 024028, [arXiv:gr-qc/0007031].

[49] Luo, Z.-X., B. Zhang, J.-J. Wei, \& X.-F. Wu, Testing Einstein's Equivalence Principle with supercluster Laniakea's gravitational field, Journal of High Energy Astrophysics 9 (2016) 35, [arXiv:1604.02566].

[50] Tully, R. B., H. Courtois, Y. Hoffman, \& D. Pomarède, The Laniakea supercluster of galaxies, Nature 513 (2014) 71, [arXiv:1409.0880].

[51] Lynden-Bell, D., S. M. Faber, D. Burstein, R. L. Davies, A. Dressler, R. J. Terlevich, et al., Spectroscopy and photometry of elliptical galaxies. V - Galaxy streaming toward the new supergalactic center, ApJ 326 (1988) 19.

[52] Adrián-Martínez, S., A. Albert, I. A. Samarai, M. André, M. Anghinolfi, G. Anton, et al., Search for muon neutrinos from gamma-ray bursts with the ANTARES neutrino telescope using 2008 to 2011 data, A\&A 559 (2013) A9, [arXiv:1307.0304].

[53] Aartsen, M. G., M. Ackermann, J. Adams, J. A. Aguilar, M. Ahlers, M. Ahrens, et al., Observation of High-Energy Astrophysical Neutrinos in Three Years of IceCube Data, Phys. Rev. Lett. 113 (2014) 101101, [arXiv:1405.5303].

[54] Abbasi, R., Y. Abdou, T. Abu-Zayyad, M. Ackermann, J. Adams, J. A. Aguilar, et al., An absence of neutrinos associated with cosmic-ray acceleration in $\gamma$-ray bursts, Nature 484 (2012) 351, [arXiv:1204.4219].

[55] Aartsen, M. G., M. Ackermann, J. Adams, J. A. Aguilar, M. Ahlers, M. Ahrens, et al., Search for Prompt Neutrino Emission from Gamma-Ray Bursts with IceCube, ApJL 805 (2015) L5, [arXiv:1412.6510]. 